Check for updates

Cite this: RSC Adv., 2019, 9, 30541

Received 3rd September 2019

Accepted 9th September 2019

DOI: 10.1039/c9ra07055a

rsc.li/rsc-advances

\section{Synthesis and characterization of rigid [2.2] paracyclophane-porphyrin conjugates as scaffolds for fixed-distance bimetallic complexes $\uparrow$}

\author{
Daniel M. Knoll, (D) $\ddagger^{a}$ Thomas B. Wiesner, $t^{a}$ Stefan M. Marschner, ${ }^{a}$ Zahid Hassan, ${ }^{a}$ \\ Patrick Weis, (D b Manfred Kappes, ${ }^{\text {be }}$ Martin Nieger ${ }^{c}$ and Stefan Bräse (iD *ad
}

This work presents a new approach to prepare mono- and disubstituted linear rigid bimetallic [2.2] paracyclophane-porphyrin conjugates via palladium-mediated Stille cross-coupling reaction. The metalated porphyrin moiety can be varied allowing convenient access to modular metal-metal fixeddistance $\mathrm{Cu} / \mathrm{Zn}$ complexes.
Catalysis is a key concept in chemistry that allows conducting reactions efficiently, using milder conditions. ${ }^{1}$ In homogeneous catalysis, metal-catalysed reactions traditionally comprise one catalyst with one transition metal centre that activates one substrate. This paradigm has been pursued for several decades. More recently, alternate catalytic approaches are being explored. They promise to allow access to difficult or otherwise unachievable reactions by for example activating two or more substrates (synergistic catalysis) ${ }^{2}$ or using two metals to activate one substrate (bimetallic catalysis). ${ }^{3}$ To understand the interplay between metal centres when introducing two or more catalytic sites in one molecule, fundamental research on cooperativity is needed. This work proposes a molecular platform for the investigation of fixed-distance metal-metal interactions relying on metalated-porphyrins and [2.2]paracyclophane as building blocks.

Because of their rigid and planar geometry, wide spectral range, absorption and emission properties, strong aromaticity and rich metal coordination chemistry, porphyrins have been extensively investigated for their vast applicability ranging from photodynamic therapy (PDT), ${ }^{4}$ mimicking enzymes, ${ }^{5}$ metal sensing, ${ }^{6}$ to organic photovoltaics. ${ }^{7,8}$ To gain access to tailor-

anstitute of Organic Chemistry, Karlsruhe Institute of Technology (KIT), Fritz-Haber-Weg 6, 76131 Karlsruhe, Germany. E-mail: braese@kit.edu

${ }^{b}$ Institute of Physical Chemistry, Karlsruhe Institute of Technology (KIT), Fritz-HaberWeg 2, 76131 Karlsruhe, Germany

'Department of Chemistry, University of Helsinki, P. O. Box 55 (A. I. Virtasen Aukio 1), ooo14 Helsinki, Finland

${ }^{d}$ Institute of Toxicology and Genetics, Karlsruhe Institute of Technology (KIT), Hermanvon-Helmholtz-Platz 1, 76344 Eggenstein-Leopoldshafen, Germany

'Institute of Nanotechnology, Karlsruhe Institute of Technology, Herman-vonHelmholtz-Platz 1, 76344 Eggenstein-Leopoldshafen, Germany

$\dagger$ Electronic supplementary information (ESI) available: Cif-files for 3a. CCDC 1877701 (3a). For ESI and crystallographic data in CIF or other electronic format see DOI: 10.1039/c9ra07055a

$\ddagger$ These two authors contributed equally to this work. made porphyrin derivatives, which are necessary for specific applications, rational design and targeted functionalization have been applied systematically. ${ }^{9-14}$ The porphyrin core, a tetradentate ligand, can coordinate to a large range of transition metal ions and form stable metal complexes which constitute useful synthetic intermediates for diverse applications. ${ }^{15,16}$ These complexes are facile in preparation and can change their photophysical properties if the metal is influenced by e.g., redox state variation, thus making porphyrins suitable scaffolds for the study of metal-metal interactions. ${ }^{17}$

[2.2]Paracyclophane (PCP) was chosen as backbone due to its rigidity and the possibility to precisely control the distance of the metal centres through exploiting its regioselective substitution/functionalization pattern (Fig. 1). PCP consists of two co-facially stacked, strongly interacting benzene rings with an average ring-to-ring distance of $3.09 \AA$. Furthermore the unique electronic structure of the PCP backbone allows through bond as well as through space $\pi-\pi$ interactions of the substituents because of the close proximity of the co-

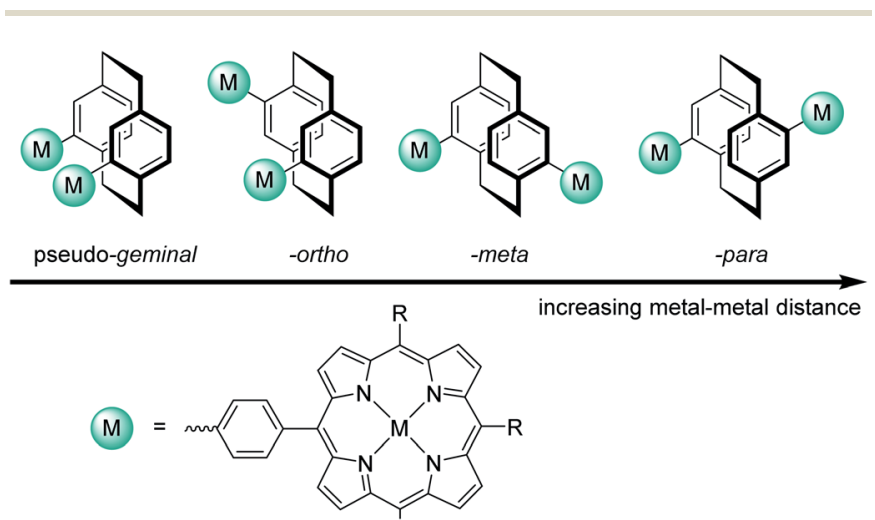

Fig. 1 The metal-metal distance can be controlled by using different substitution patterns on the [2.2] paracyclophane. 
facially stacked benzene rings and resulting $\pi$-system overlapping. ${ }^{\mathbf{1 8}}$

Metalated porphyrins linked to [2.2]paracyclophanes prepared by condensation of PCP-carbaldehyde and pyrrole have been described in literature. ${ }^{19-21} \mathrm{~A}$ theoretical study to understand the importance of through-space interactions and the rather strong effects of a PCP moiety in zinc-porphyrin molecular wires and their comparison to oligo( $p$-phenylenevinylene) for photovoltaic applications have been investigated. ${ }^{22,23}$ Furthermore, Malinski et al. reported the formation of a directly linked conjugate from a mixed condensation in low yields requiring tedious workup. ${ }^{21}$ Martin et al. formed a vinylic bridge between PCP and porphyrin via a Horner-WadsworthEmmons reaction in about $40 \%$ yield. ${ }^{\mathbf{1 4}}$ However, a linear linker would allow for a more careful planning of the molecular geometry while exploiting the high rigidity of [2.2]paracyclophane. ${ }^{24}$ The facile variation of the metal coordinated to the porphyrin moiety and the possibility of tuning the distance between the metal centres using the different [2.2]paracyclophane regioisomers make this ensemble a suitable scaffold for assessing modular fixed-distance bimetallic complexes.

Since the previously reported optimization of reaction conditions to achieve this did not lead to satisfying yields, ${ }^{21}$ a different approach was pursued in this study.

The Stille cross-coupling reaction protocol was shown to be applicable to both, porphyrins and [2.2]paracyclophanes. ${ }^{25,26}$ The reaction parameters including palladium source, temperature, and solvent were carefully optimized.

The use of free-base porphyrin 2a and (rac)-4-stannyl[2.2] paracyclophane $\mathbf{1}$, which was synthesized according to a literature known procedure starting from ( $r a c)-4$-bromo[2.2]paracyclophane, ${ }^{26,27}$ resulted in the formation of the copper-complex $3 \mathbf{a}$ in $20 \%$ yield (Scheme 1). The copper(II)-ions are assumed to originate from impurities of $\mathrm{CuBr}_{2}$ in the used $\mathrm{CuBr}$ or from an undesired oxidation side reaction. The absence of the free-base product indicates that the reaction only proceeds with metalated porphyrins and the free-base porphyrin remains inert under these conditions. Therefore, protection of the porphyrin core with $\mathrm{Cu}$ (II)-ions (2b) or $\mathrm{Zn}$ (II)-ions (2c) was performed, ${ }^{25}$ leading to an increase of the yield of the respective resulting coupling products up to 48 and $52 \%$ (Scheme 1). The molecular structure of compound $3 \mathrm{a}$ was unambiguously confirmed by $\mathrm{X}$ - ray single crystal analysis (Fig. 2) and further characterized using collision induced dissociation (CID) experiments in ESI mass spectrometry (see ESI $\dagger$ ).

In a further approach the functionalities of the two coupling reactants as described in Scheme 1 were mutually exchanged to investigate the comparative reactivity and improve yields, by reacting ( $r a c$ )-4-bromo[2.2]paracyclophane with the corresponding zinc containing stannyl-porphyrin 6 as nucleophilic reacting partner. The reaction gave $\mathbf{3} \mathbf{b}$ with a similarly high yield of $41 \%$.

After successful demonstration of mono-functionalization, disubstitution using the bifunctionalized [2.2]paracyclophane derivatives 4 and $\mathbf{5}$ was attempted (Scheme 2). Purification of the distannyl [2.2]paracyclophane 4 proved to be challenging and the crude reaction product was used in the synthesis of the pseudo-para disubstituted derivative 7 . The impurities that remained are most likely the reason for the significant drop in yield in the reaction with brominated porphyrin $2 \mathrm{c}$. The desired product was obtained in a yield of $2 \%$ and serves as a first proof

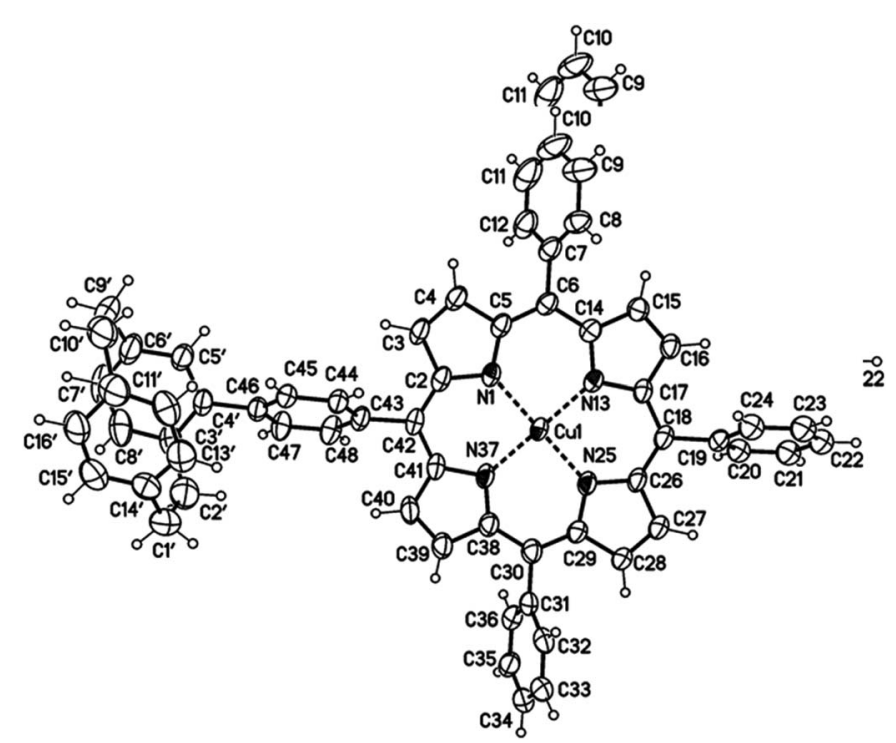

Fig. 2 Molecular structure of the paracyclophane-porphyrin conjugate 3a (minor disordered part omitted for clarity, displacement parameters are drawn at $50 \%$ probability level; details see ESI†).
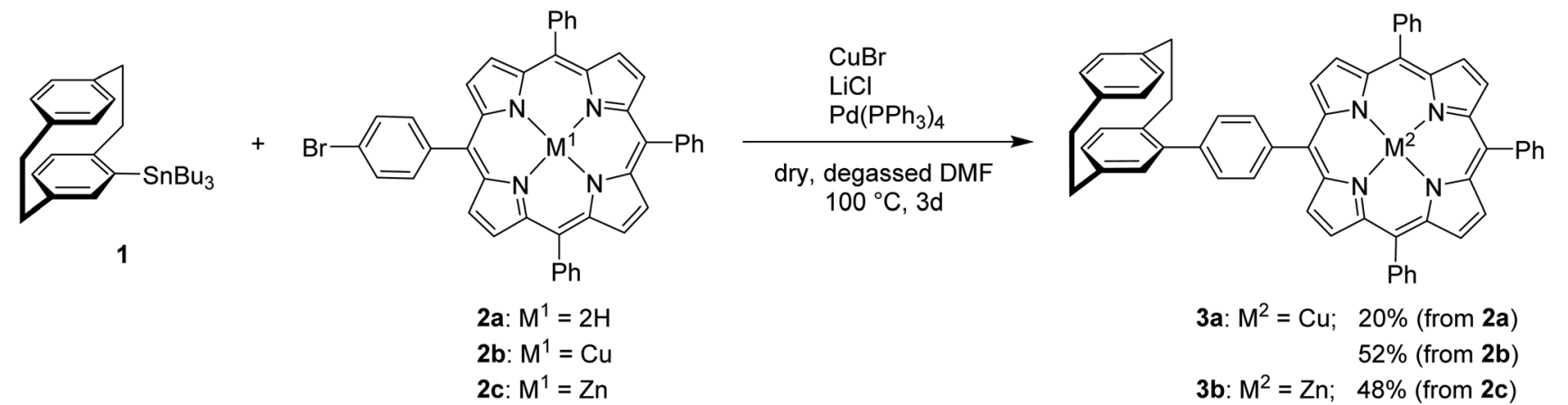

Scheme 1 Synthesis of [2.2] paracyclophane-porphyrin conjugates linked by a phenyl unit via Stille cross-coupling protocol. 

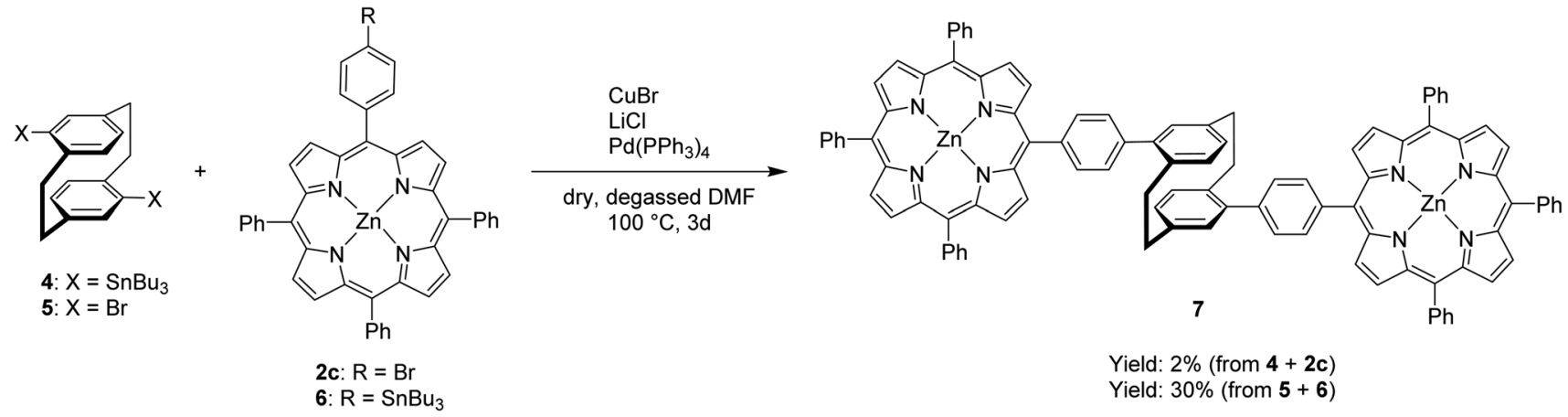

Yield: $2 \%$ (from $4+2 c)$

Yield: $30 \%($ from $5+6$ )

Scheme 2 Synthesis of (rac)-4,16-di-(4-((10,15,20-triphenylporphyrin)zinc(II))phenyl) [2.2]paracyclophane(7)

of concept. The functionalities of the reactants were exchanged again, in order to use compounds which are easier to handle. When the porphyrin stannane 6 and the pseudo-para dibromo [2.2]paracyclophane $\mathbf{5}$, which was synthesized according to a literature known procedure, ${ }^{28}$ were used, the yield increased up to $30 \%$. The bisporphyrin 7 was characterized via mass spectrometry. Methanol and formic acid needed to be added to the solution in DCM before the compound could be detected with the nano-ESI method in positive mode, because of its low polarity and difficult ionization.

This new class of modular compounds might serve a molecular platform for the investigations of fixed-distance metalmetal interactions, held together by a rigid [2.2]paracyclophane. To access enantiomerically pure mono- and disubstituted PCP derivatives, various procedures of chiral resolution using optimum chiral auxiliaries, for instance, derivatives of L-amino acids, (+)-naproxen, (S)-(-)-camphanoyl chloride, $(S)-(+)-10-$ camphorsulfonic acid, (-)-menthol and others have been reported as promising methodology towards a wide range of precursors in an enantiopure form. ${ }^{29-33}$ Synthesis of homo- and heterobimetallic pseudo-ortho, -meta and -geminal derivatives of this class and screening of different metals for catalytic activities is currently underway and we believe that may serve to highlight the immense potential for the study of intermetallic cooperative effects in complexes.

\section{Conflicts of interest}

There are no conflicts to declare.

\section{Acknowledgements}

The authors thank the Deutsche Forschungsgemeinschaft (DFG) for the support of this work through the collaborative research center SFB/TRR 88 “3MET" (Cooperative Effects in homo- und heterometallic complexes), projects B2 and C6.

\section{Notes and references}

1 K. P. C. Vollhardt, and N. E. Schore, Organic Chemistry; Palgrave Version: Structure and Function, Macmillan International Higher Education, 2014.
2 A. E. Allen and D. W. MacMillan, Chem. Sci., 2012, 3, 633658.

3 D. R. Pye and N. P. Mankad, Chem. Sci., 2017, 8, 1705-1718. 4 J. P. Celli, B. Q. Spring, I. Rizvi, C. L. Evans, K. S. Samkoe, S. Verma, B. W. Pogue and T. Hasan, Chem. Rev., 2010, 110, 2795-2838.

5 S. L. Rebelo, M. Linhares, M. M. Simões, A. M. Silva, M. G. P. Neves, J. A. Cavaleiro and C. Freire, J. Catal., 2014, 315, 33-40.

6 D. Vlascici, E. Fagadar-Cosma, E. Pica, V. Cosma, O. Bizerea, G. Mihailescu and L. Olenic, Sensors, 2008, 8, 4995-5004.

7 M. R. Wasielewski, Acc. Chem. Res., 2009, 42, 1910-1921.

8 D. Gust, T. A. Moore and A. L. Moore, Acc. Chem. Res., 2009, 42, 1890-1898.

9 M. O. Senge and X. Feng, J. Chem. Soc., Perkin Trans. 1, 2000, 3615-3621.

10 M. O. Senge, Chem. Commun., 2011, 47, 1943-1960.

11 P. D. Rao, S. Dhanalekshmi, B. J. Littler and J. S. Lindsey, J. Org. Chem., 2000, 65, 7323-7344.

12 S. Plunkett and M. O. Senge, ECS Trans., 2011, 35, 147-157.

13 S. Hiroto, Y. Miyake and H. Shinokubo, Chem. Rev., 2016, 117, 2910-3043.

14 J. S. Lindsey, Acc. Chem. Res., 2009, 43, 300-311.

15 J. L. Sessler, Z. Gross and H. Furuta, Chem. Rev., 2017, 117, 2201-2202.

16 M. Kielmann and M. O. Senge, Angew. Chem., Int. Ed., 2019, 58, 418-441.

17 J. P. Collman, C. M. Elliott, T. R. Halbert and B. S. Tovrog, Proc. Natl. Acad. Sci., 1977, 74, 18.

18 M. Wielopolski, A. Molina-Ontoria, C. Schubert, J. T. Margraf, E. Krokos, J. Kirschner, A. Gouloumis, T. Clark, D. M. Guldi and N. Martín, J. Am. Chem. Soc., 2013, 135, 10372-10381.

19 L. Czuchajowski and M. Lozynski, J. Heterocycl. Chem., 1988, 25, 349-350.

20 S. Banfi, A. Manfredi, F. Montanari, G. Pozzi and S. Quici, J. Mol. Catal. A: Chem., 1996, 113, 77-86.

21 L. Czuchajowski, J. Bennett, S. Goszczynski, D. Wheeler, A. Wisor and T. Malinski, J. Am. Chem. Soc., 1989, 111, 607-616.

22 Y. Kobayashi, T. Katayama, T. Yamane, K. Setoura, S. Ito, H. Miyasaka and J. Abe, J. Am. Chem. Soc., 2016, 138, 59305938. 
23 Y. Orimoto, K. Kato and Y. Aoki, J. Phys. Chem. C, 2017, 121, 17703-17711.

24 Z. Hassan, E. Spuling, D. M. Knoll, J. Lahann and S. Bräse, Chem. Soc. Rev., 2018, 47, 6947-6963; Z. Hassan, E. Spuling, D. M. Knoll and S. Bräse, Angew. Chem., Int. Ed., 2019, DOI: 10.1002/anie.201904863.

25 N. N. Sergeeva, A. Scala, M. A. Bakar, G. O'Riordan, J. O'Brien, G. Grassi and M. O. Senge, J. Org. Chem., 2009, 74, 7140-7147.

26 C. Braun, E. Spuling, N. B. Heine, M. Cakici, M. Nieger and S. Bräse, Adv. Synth. Catal., 2016, 358, 1664-1670.

27 D. J. Cram and A. C. Day, J. Org. Chem., 1966, 31, 1227-1232.
28 H. J. Reich and D. J. Cram, J. Am. Chem. Soc., 1969, 91, 35343543.

29 G. J. Rowlands and R. J. Seacome, Beilstein J. Org. Chem., 2009, 5, 9.

30 R. Parmar, M. P. Coles, P. B. Hitchcock and G. J. Rowlands, Synthesis, 2010, 4177-4187.

31 R. Zhuravsky, Z. Starikova, E. Vorontsov and V. Rozenberg, Tetrahedron: Asymmetry, 2008, 19, 216-222.

32 Y. Morisaki, R. Hifumi, L. Lin, K. Inoshita and Y. Chujo, Chem. Lett., 2012, 41, 990-992.

33 Y. Morisaki, M. Gon, T. Sasamori, N. Tokitoh and Y. Chujo, J. Am. Chem. Soc., 2014, 136, 3350-3353. 